\title{
Parásitos intestinales en poblaciones ferales de palomas domésticas (Columba livia domestica) en Coro, estado Falcón, Venezuela
}

\author{
Intestinal parasites in feral populations of domestic pigeons (Columba livia \\ domestica) in Coro, Falcon State, Venezuela
}

Dalmiro Cazorla Perfetti ${ }^{1,2}$, Pedro Morales Moreno ${ }^{1}$

\section{Resumen}

\begin{abstract}
La paloma doméstica (Columba livia domestica) es una especie de ave columbiforme muy bien adaptada a los ambientes intervenidos por el ser humano. Sin embargo, puede servir de transportador de microorganismos patógenos de interés médico-zoonótico. Entre agosto de 2017 y agosto de 2018 se recolectaron heces de poblaciones ferales de palomas domésticas para determinar la ocurrencia de parásitos intestinales en tres sitios de la ciudad de Coro, estado Falcón, Venezuela: en el Hospital Universitario «Alfredo Van Grieken», en el terminal de pasajeros municipal y en la Universidad Nacional Experimental «Francisco de Miranda». Las muestras $(n=516)$ se analizaron mediante cinco métodos coprológicos: directo, flotación de Willis-Molloy, flotación de Faust, sedimentación en tubo y coloración de Kinyoun. El análisis parasitológico reveló la presencia en el 54.3\% (280/516) de las muestras fecales de 12 taxones enteroparasitarios, de las cuales varias son consideradas potencialmente de importancia médico-zoonótico (Criptosporidium spp, Blastocystis spp, Cyclospora spp). Los taxones más prevalentes fueron Cryptosporidium spp (38.5\%), Isospora spp (19.4\%), Cyclospora spp (13\%) y Raillietina spp (7.8\%). Se discute las implicaciones de estos hallazgos en el contexto de la salud pública.
\end{abstract}

Palabras clave: Columba livia domestica; parásitos intestinales; Venezuela; Criptosporidium

\footnotetext{
${ }^{1}$ Laboratorio de Entomología, Parasitología y Medicina Tropical (L.E.P.A.M.E.T.), Centro de Investigaciones Biomédicas (C.I.B.), Universidad Nacional Experimental «Francisco de Miranda» (UNEFM), Estado Falcón, Venezuela

${ }^{2}$ E-mail: lutzomyia@hotmail.com
}

Recibido: 8 de septiembre de 2018

Aceptado para publicación: 15 de abril de 2019 
The domestic pigeon (Columba livia domestica) is a columbiform bird species welladapted to anthropic environments. However, it can serve as a carrier of pathogenic microorganisms of zoonotic interest. Between August 2017 and August 2018 feces of feral populations of domestic pigeons were collected to determine the occurrence of intestinal parasites in three sites of the city of Coro, Falcon state, Venezuela: University Hospital «Alfredo Van Grieken», a bus terminal and the National Experimental University «Francisco de Miranda». The samples $(\mathrm{n}=516)$ were analyzed by five coprological methods: direct test, Willis-Molloy flotation ( $\mathrm{ClNa}$ ), Faust flotation (zinc sulphate), spontaneous sedimentation in tube and Kinyoun staining. The parasitological analysis revealed the presence of 12 parasitic taxa in $54.3 \%$ (280/516) of faecal samples, of which several are considered of medical-zoonotic importance (Cryptosporidium spp, Blastocystis spp, Cyclospora spp). The most prevalent taxa were Criptosporidium spp (38.5\%), Isospora spp (19.4\%), Cyclospora spp (13\%) and Raillietina spp (7.8\%). The implications of these findings in the context of public health are discussed.

Key words: Columba livia domestica; intestinal parasites; Venezuela; Criptosporidium

\section{INTRODUCCIÓN}

La paloma doméstica (Columba livia domestica), especie de la familia Columbidae, puede volar grandes distancias y se encuentra bien adaptada a los ambientes sinantrópicos, desde las grandes urbes hasta las áreas rurales donde alcanzan elevadas densidades poblacionales, siendo utilizadas como fuente alimentaria, hobby o con fines experimentales (Tietz-Marques et al., 2007; Bahrami et al., 2012). Sin embargo, las palomas domésticas probablemente sean las aves que representan la mayor molestia para los seres humanos, llegando a convertirse en plagas urbanas (Tietz-Marques et al., 2007; Bahrami et al., 2012; De La Ossa et al., 2017).

Las palomas anidan en cualquier saliente de casas, edificios, puentes, etc, causando daños a las estructuras y repulsión por sus sonidos y heces, orines y polvo de sus plumas; además, pueden transportar, transmitir o ser reservorios de una amplia variedad de microrganismos y artrópodos potencialmente patógenos para el ser humano y otros animales (Tietz-Marques et al., 2007; Tanveer et al., 2011; Bahrami et al., 2012; De La Ossa et al., 2017). Dentro de las afecciones más conocidas se encuentran la histoplasmosis, la enfermedad de Newcastle, psitacosis u ornitosis, criptococcosis, coccidiosis, toxoplasmosis, pseudotuberculosis y salmonelosis (Tietz-Marques et al., 2007; Tanveer et al., 2011; Bahrami et al., 2012; Badparva et al., 2015).

Las palomas domésticas se alimentan de una gran variedad de fuentes alimentarias, incluyendo granos y una serie de invertebrados, los cuales pueden contener estadios de parásitos infectivos (Diakou et al., 2013). Dentro de los microorganismos entero-parasitarios de importancia zoonótica que afectan a las palomas domésticas, resaltan los cromistas, agentes causantes de diarrea aguda y crónica prolongada, como Cryptosporidium spp (criptosporidiosis) y Blastocystis spp (blastocistosis) (Hrdlicková, 2011; Botero y Restrepo, 2012; Koompapong et al., 2014).

La bibliografía del área para Venezuela es escasa y antigua, y no se ha enfatizado en especies entero-parasitaria de interés para la 
salud pública (Díaz-Ungría, 1981). Como en la gran mayoría de los centros urbanos de Venezuela, en la ciudad de Coro, enclavada en la zona semiárida del estado Falcón, en la región nor-occidental de Venezuela, y sus alrededores, la presencia de grandes poblaciones de palomas domésticas es un hecho vivencial y común. Es por ello que el presente tuvo como objetivo investigar los taxones enteroparasitarios presentes en muestras fecales de poblaciones de palomas domésticas ferales en la ciudad de Coro.

\section{Materiales y Métodos}

El estudio se llevó a cabo entre enero de 2017 y agosto de 2018 en la ciudad de Coro, capital del estado Falcón, en la región semiárida noroccidental de Venezuela, cuyas características bioclimáticas han sido reseñadas en una publicación previa (Cazorla y Morales, 2013).

Se escogieron tres sitios de la ciudad de Coro que presentaban poblaciones ferales de palomas domésticas: el Hospital Universitario «Alfredo Van Grieken» (HUAV), el terminal de pasajeros municipal «Polica Salas» (TPM) y el área Ciencias de la Salud de la Universidad Nacional Experimental «Francisco de Miranda» (UNEFM). En cada salida de campo (06:00-07:00) se recolectaron en envases estériles herméticos entre 5-10 muestras fecales frescas de palomas en puntos equidistantes de 2.5-3.0 m. Se colectaron en total 516 muestras (HUAV=209; $\mathrm{TPM}=157$; UNEFM=150), que fueron llevadas en cavas de poliuretano al Laboratorio de Entomología, Parasitología y Medicina Tropical (LEPAMET) del Centro de Investigaciones Biomédicas (CIB) de la UNEFM. Las muestras, de no ser analizadas el día de la toma, se preservaron y fijaron añadiéndoles $1-2 \mathrm{ml}$ de formaldehido al $10 \%$.

El diagnóstico coproparasitológico se hizo con cinco métodos, incluyendo el directo y las técnicas de ûotación simple de Willis-
Molloy con solución sobresaturada de cloruro de sodio $(\mathrm{NaCl})$, ûotación-centrifugación de Faust con sulfato de zinc ( $\mathrm{ZnSO} 4$ ), de sedimentación en tubo (TSET) y coloración de Kinyoun (Ziehl-Neelsen modificado) (Morales y Pino de Morales, 1977; Botero y Restrepo, 2012). Las muestras fecales se cultivaron a temperatura ambiente por 2-5 días en una solución de dicromato de potasio $(2.5 \%)$ para diferenciar los ooquistes no esporulados de coccidios de Isospora spp y Eimeria spp. Las heces se observaron y fotografiaron (Olympus, Fe-120, Olympus Imaging Corp., Japón) por duplicado bajo microscopio de luz (Axiostar Plus, Carl Zeiss, Alemania), con y sin solución yodada de lugol. Las muestras se consideraron con presencia de parásitos cuando se detectaba al menos un estadio (quiste/ooquistes/huevo/larva) de protozoario/cromista o helminto.

Ante la posibilidad de que algunas de las muestras de heces pudieron haberse originado de la misma ave (Cazorla y Morales, 2017), se decidió utilizar el término «ocurrencia» en vez de «prevalencia» para describir el porcentaje de positivos; asimismo, a la ocurrencia se le determinó su intervalo de confianza (IC 95\%).

Se calculó el índice de diversidad de riqueza específica $(S)$ y el de dominancia de Simpson $(\lambda)$, para estimar las diferencias entre la composición de las comunidades de parásitos intestinales de las palomas en los tres ambientes estudiados. El índice $S$ aporta el número total de especies obtenido en el muestreo; el índice $\lambda$ varía desde 0 (todos los taxones se encuentran igualmente presentes) a 1 (un taxón domina la comunidad completamente) (Hammer et al., 2001; Moreno, 2001). La posible asociación entre la distribución de los taxones enteroparasitarios y los diferentes ambientes muestreados se hizo mediante un Análisis de Correspondencia (Greenacre, 2008). Los datos se analizaron mediante el paquete estadístico PAST v. 3.18 (Hammer et al., 2001). 


\section{Resultados y Discusión}

La ocurrencia global de parásitos intestinales fue del $54.3 \%$ (IC: $49.7-58.3 \%$ ) (280/516). La ocurrencia de los diversos taxones de helmintos y cromistas se presenta en el Cuadro 1. Dentro de los cromistas, los coccidios de los géneros Cryptosporidium spp (38.5\%) e Isospora spp (19.4\%) fueron los taxones más frecuentemente detectados, mientras que el cestodo Raillietina spp (7.8\%) y un trematodo no identificado (7.2\%) fueron los taxones de helmintos que presentaron las mayores ocurrencias en las muestras fecales (Cuadro 1; Figuras 1 y 2). El monoparasitismo se presentó en el 30.4\% $(85 / 280)$ de las heces positivas a parásitos, mientras que en el caso de las infecciones múltiples se detectaron muestras hasta con cinco taxones de parásitos.

El índice $S$ de la fauna parasitaria e intervalo de confianza (95\%) fue de 9 (7-9), 9 (9-9), 9 (9-9) para los sitios de captura UNEFM, HUAV y TPM, respectivamente, mientras que el índice de abundancia (ë) fue de 0.28 (0.24-0.34), 0.22 (0.2-0.26), 0.15 (0.14-0.18) para UNEFM, HUAV y TPM, respectivamente. Los resultados muestran una distribución más uniforme de los individuos entre las especies enteroparasitarias.

Con la aplicación del Análisis de Correspondencia solo se evidenció una acentuada asociación entre los taxones enteroparasitarios Hymenolepis spp, Tetrameres spp y el nematodo no identificado con el recinto de educación universitaria (UNEFM) (Figura 3).

El hallazgo en el presente estudio de una ocurrencia global mayor a $50 \%$ de parásitos intestinales, incluyendo varios taxones de interés zoonótico en las muestras fecales de poblacionales ferales de palomas domésticas, revela su importancia potencial sanitaria para los habitantes de Coro. Las cifras de ocurrencia/prevalencia de parasitosis intestinales en $C$. livia domestica pueden variar de acuerdo a varios factores (e.g., época del año, métodos de diagnóstico, factores climáticos, eco-geográficos); así, la tasa de ocurrencia enteroparasitaria de $54.3 \%$ registrada en Coro es mayor a las detectadas en Egipto (46.2\%) (Nagwa et al. 2013) e Irán (42.2\%) (Radfar et al., 2011), pero menores a las encontradas en India (75\%) (Jayentakumar Singh y Mohilal, 2017), Brasil (75\%) (Tietz-Marques et al., 2007) y en otro estudio en Irán (89.6\%) (Bahrami et al. 2012).

Dentro de las coccidiosis que afectan comúnmente a las palomas domésticas, destacan la eimeriosis y la isosporiosis ocasionadas por los coccidios patógenos Eimeria spp e Isospora spp, respectivamente. De acuerdo con las fuentes consultadas, en estas aves se ha descrito una especie de Isospora spp y nueve de Eimeria spp (Balicka-Ramisz y Pilarczyk, 2014), siendo reportada $E$. labbeana en C. livia domestica para Venezuela (Díaz-Ungría, 1981). Estos coccidios pueden ocasionar diarrea con sangre y debilitamiento con pérdida de peso en las aves, lo que las hace más susceptibles a otras infecciones y, potencialmente la muerte, especialmente en las palomas de menor edad en un 5-30\% de los casos (Krautwald-Junghanns et al., 2009; Balicka-Ramisz y Pilarczyk, 2014). La tasa de ocurrencia de 3.9\% para Eimeria spp detectada en el presente estudio es menor a las obtenidas en la mayoría de las investigaciones hechas en otras regiones del mundo (21.6-100\%) (Tietz-Marques et al., 2007; Sari et al. 2008; Krautwald-Junghanns et al., 2009; Radfar et al., 2011; Bahrami et al. 2012; Nagwa et al. 2013; Balicka-Ramisz y Pilarczyk, 2014; Pérez-García et al. 2015); mientras que la tasa de $19.4 \%$ para Isospora spp se encuentra dentro del rango de frecuencia de 18.4-70\% (Sari et al. 2008; Jayentakumar Singh y Mohilal, 2017).

Aun teniendo en consideración que se implementó la técnica de Kinyoun sin el complemento de otras herramientas diagnósticas como la detección de coproantígenos o PCR, el $38.5 \%$ de positividad detectado para 
Cuadro 1. Frecuencia absoluta y ocurrencia (\%) de parásitos intestinales en heces $(\mathrm{n}=516)$ de palomas domésticas en Coro, estado Falcón, Venezuela (2017-2018)

\begin{tabular}{lccc}
\hline \multirow{2}{*}{ Taxón } & \multicolumn{3}{c}{ Muestras positivas } \\
\cline { 2 - 4 } & $(\mathrm{n})$ & $(\%)$ & $\begin{array}{c}\text { Intervalo de } \\
\text { confianza (95\%) }\end{array}$ \\
\hline Cromistas & & & \\
Blastocystis spp & 38 & 7.4 & $5.1-9.7$ \\
Cyclospora spp & 67 & 13 & $10.1-16.0$ \\
Eimeria spp & 20 & 3.9 & $2.2-5.6$ \\
Isospora spp & 100 & 19.4 & $15.6-22.4$ \\
Cryptosporidium spp & 185 & 35.9 & $31.9-40.1$ \\
Helmintos & & & \\
$\quad$ Capillaria spp & 41 & 8 & $5.7-10.3$ \\
Syngamus spp & 11 & 2.1 & $0.9-3.3$ \\
Raillietina spp & 40 & 7.8 & $5.5-10.1$ \\
Tetrameres spp & 2 & 0.4 & $0.2-1.0$ \\
Trematodo no identificado & 37 & 7.2 & $5.0-9.4$ \\
Hymenolepis spp & 1 & 0.2 & $0.2-0.6$ \\
Nematodo no identificado & 1 & 0.2 & $0.2-0.6$ \\
\hline Total & 280 & 54.3 & $49.7-58.3$ \\
\hline
\end{tabular}

Cryptosporidium spp en muestras fecales de palomas en Coro es mayor [Perú: 29\%, Vargas (2016); Iraq: 26.7\%, Jasim y Marhoon (2015); España: 5.9\%, Abreu-Acuña et al. (2009); Irán: 1.2-2.9\%, Radfar et al. (2011); Egipto: 12.1\%, Nagwa et al. (2013); China: 0.82\%, Li et al. (2015a); Brasil: 4-7\%, Oliveira et al. (2017); 0\%: Italia (Marenzoni et al., 2016), Turquía (Sari et al., 2008) y Colombia (Pérez-García et al., 2015)], menor [Perú: 46\%, Copia y Quiroga (2017)] o similar [Iraq: 40\%, Faraj (2014)] a las cifras encontradas en otras regiones del mundo.

El presente trabajo aparece como el primer reporte de Cryptosporidium spp en C. livia domestica para Venezuela. En las palomas domésticas se han aislado varias especies y subtipos de Cryptosporidium spp, incluyendo $C$. parvum, $C$. meleagridis, $C$. hominis, C. baileyi y C. galli, las que pue- den tener efectos patológicos graves en las aves, incluyendo a C. livia domestica (Wang et al., 2011; Santín, 2013). Si se resalta que las cuatro primeras especies mencionadas también han sido detectadas en el humano ocasionando efectos patológicos de consideración, especialmente en individuos inmunocomprometidos (Ditrich et al., 1991; Wang et al., 2018), entonces existe la posibilidad de la transmisión zoonótica de este coccidio para los habitantes de Coro; de allí que se requiere tipificar molecularmente los aislados de Cryptosporidium spp de la región.

El género Cyclospora spp comprende alrededor de 19 especies que infectan una amplia variedad de hospedadores (invertebrados y vertebrados) (Lainson, 2005). Hasta el presente, el humano aparece como el único hospedador específico de C. cayetanensis, 


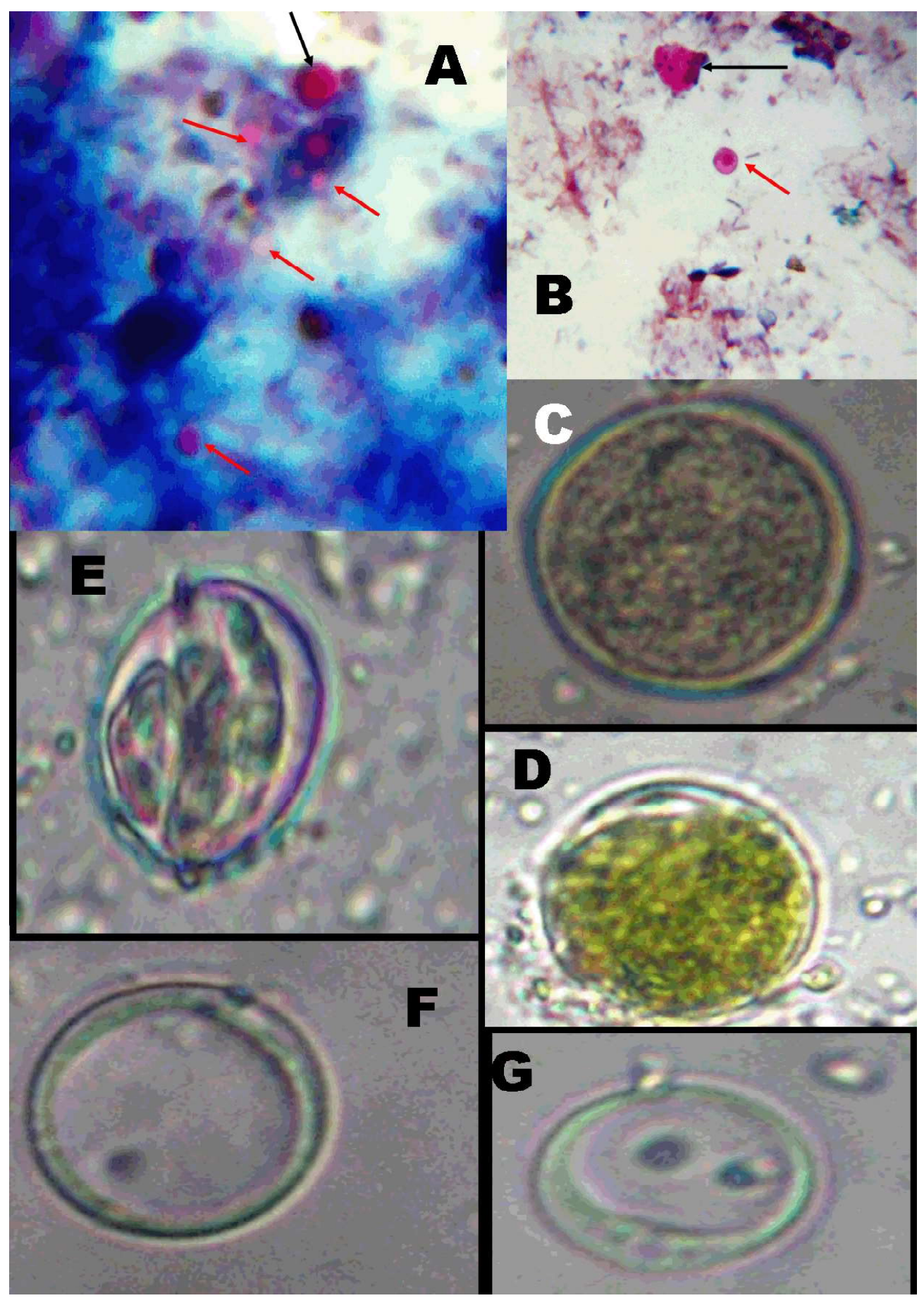

Figura 1. Taxones parasitarios aislados en muestras fecales de palomas domésticas (Columba livia domestica) en la ciudad de Coro, estado Falcón, Venezuela (2017-2018). (a, b) Ooquistes de Cryptosporidium spp (flechas rojas) y Cyclospora spp (flechas negras). Tinción Kinyoun, 1000 X; (c, d) Ooquistes de Isospora spp; (e) Ooquistes de Eimeria spp; (f, g) Quistes de Blastocystis spp. Tinción lugol, 400X 


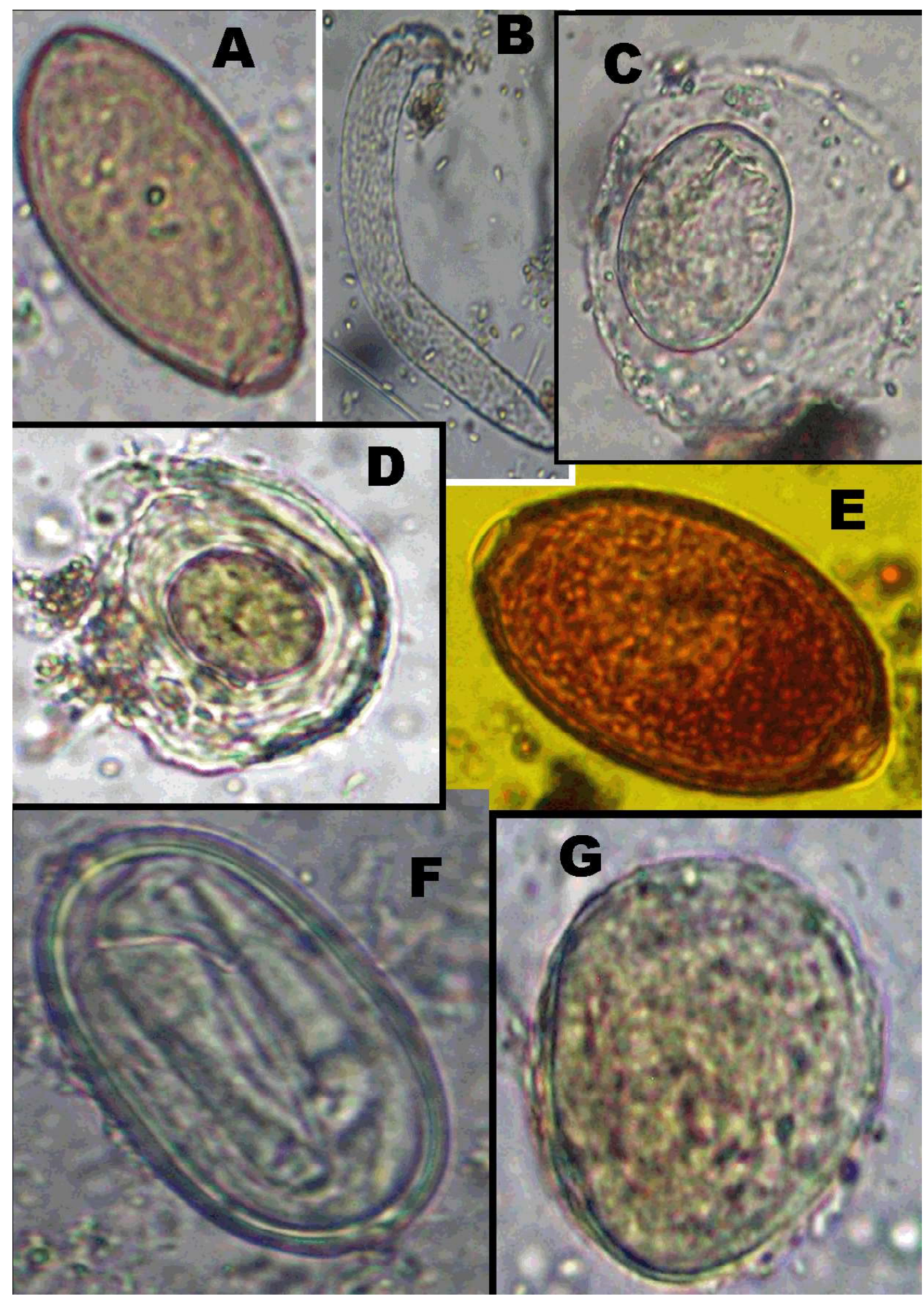

Figura 2. Taxones parasitarios aislados en muestras fecales de palomas domésticas (Columba livia domestica) en la ciudad de Coro, estado Falcón, Venezuela (2017-2018). (a) Huevo de trematodo no identificado; (b) larva de nematodo no identificado; (c) Huevo de Raillietina spp; (d) Huevo de Hymenolepis spp; (e) Huevo de Capillaria spp; (f) Huevo de Syngamus spp; (g) Huevo de Tetrameres spp. Tinción lugol, 400X 


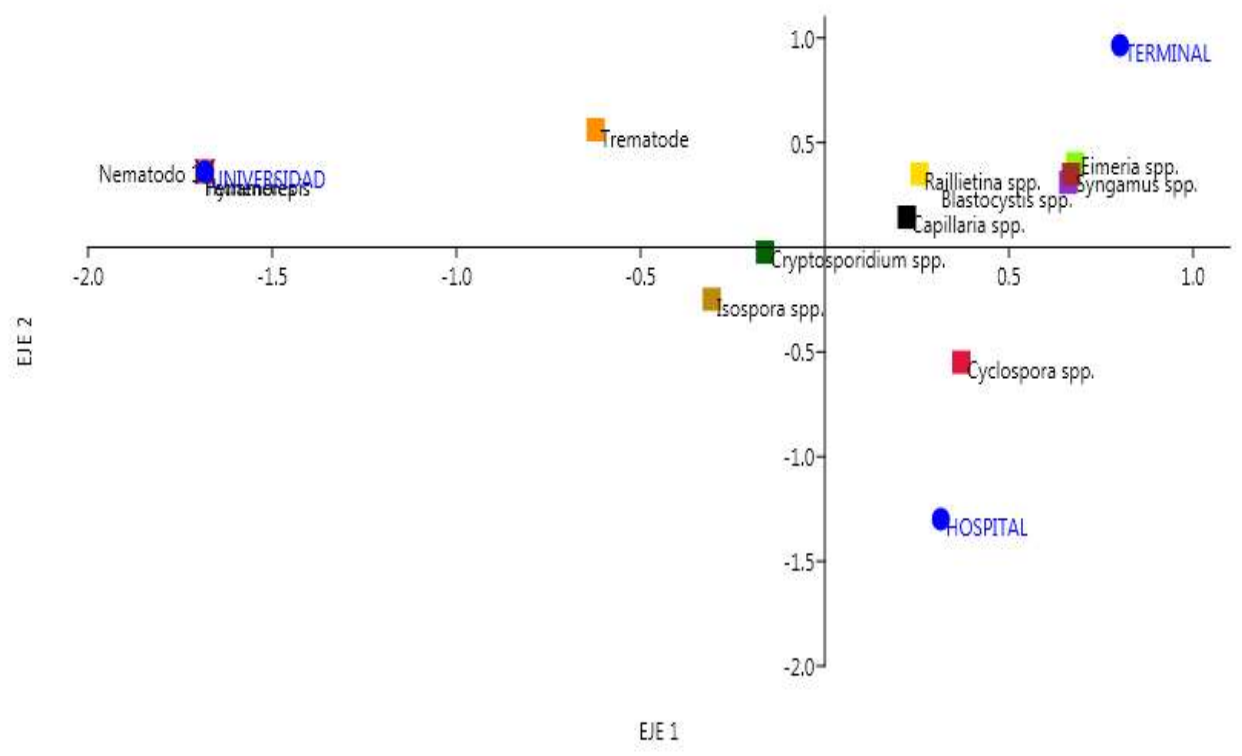

Figura 3. Asociación de los taxones enteroparasitarios aislados de palomas domésticas y los ambientes muestreados mediante Análisis de Correspondencia (Eje 1: eigenvalor $=$ $0.16 ; 61.7 \%$; Eje 2 : eigenvalor $=0.1 ; 38.3 \%$ ). Hospital: HUAV; Terminal: TPM; Universidad: UNEFM

agente causal de la denominada «diarrea del viajero», lo que se apoya con estudios histológicos donde el parásito realiza el ciclo completo en el intestino delgado (Lainson, 2005; Chacín Bonilla y Vielma, 2018). Sin embargo, sustentado mediante métodos parasitológicos convencionales y técnicas moleculares, $C$. cayetanensis ha sido detectado en varias especies de animales tanto silvestres como domésticas, incluyendo aves, pero no en $C$. livia domestica; no obstante, en ningún caso se ha demostrado el desarrollo ni la acción patógena del coccidio sobre estos animales (Chu et al., 2004; Li et al., 2015b; Chacín Bonilla y Vielma, 2018). Con base a esto, se requiere determinar la caracterización genética y patogenicidad de los ooquistes con morfología compatible con Cyclospora spp detectados en las palomas domésticas de Coro para dilucidar si estas aves son transportadores o propagadores mecánicos de estos coccidios y, por lo tanto, constituyen un factor de riesgo potencial para adquirir la ciclosporiosis.
Llama la atención los pocos reportes de Blatocystis spp en palomas domésticas; así, Hrdlièková (2011) en la República Checa solo detectó una muestra fecal positiva de 25 analizadas con métodos de cultivo y PCR. El presente estudio aparece como el primer hallazgo de Blatocystis spp en C. livia domestica en Venezuela. De los 17 subtipos moleculares (ST1 a ST17) que presenta Blastocystis spp, en palomas y aves domésticas se ha aislado el subtipo ST7 el cual junto con el subtipo ST6 se les tiene como los «ST aviares»; aunque en aves también se han detectado otros subtipos (ST2, ST5) (Hrdlièková 2011; Lewicki et al. 2016; Badparva et al. 2017; Greige et al. 2018). El hecho de que en humanos se han detectado los subtipos ST1 a ST9 (Stensvold y Clark 2016), plantea la potencialidad de transmisión zoonótica que poseen los aislamientos observados en palomas domésticas en Coro, así como también por otros autores, tanto en aves como en otros animales (Hrdlièková 2011; Stensvold y Clark 2016; Betts et al., 2018). Por ello, se requie- 
re determinar la caracterización genética de los aislamientos de Blastocystis spp que circulan tanto en palomas domésticas como en otros animales y humanos en la ciudad de Coro y el estado Falcón, donde las tasas de blastocistosis humana pueden sobrepasar el 50\% (Cazorla-Perfetti et al., 2014, 2015).

Los nematodos del género Capillaria spp son ampliamente prevalentes en aves, especialmente en Galliformes y Columbiformes incluyendo a C. livia domestica (Pazmiño, 2007; Pérez-García et al., 2015). El hallazgo de este tipo de Trichuridae en las palomas indica que los hospedadores intermediarios como las lombrices de tierra (anélidos) constituyen parte de su dieta. Las cifras de ocurrencia encontradas en las poblaciones de palomas domésticas en Coro de $8 \%$ son menores a las reportadas en Chile, Brasil, Colombia, Turquía y Pakistán (1667.2\%) (Pazmiño, 2007; Tietz-Marques et al., 2007; Sari et al., 2008; Tanveer et al., 2011; Pérez-García et al., 2015), pero inferiores a las encontradas en Irán (6\%) (Bahrami et al., 2012) e India (1.5\%) (Jayentakumar Singh y Mohilal, 2017); aunque se debe señalar que no han sido detectados en otros estudios. Los otros dos taxones de nematodos identificadas a nivel de género fueron Syngamus spp y Tetrameres spp, cuyas tasas de ocurrencia resultaron bajas $(2.1 \%$ y $0.4 \%$, respectivamente).

En relación con los trematodos o duelas, se halló una tasa de ocurrencia de $7.2 \%$ de huevos operculados que no se pudieron identificar a nivel de género. Se considera que el parasitismo por trematodos en aves Columbiformes es ocasional, al ingerir los estadios infectantes en reservorios de aguas que los contienen (Pazmiño, 2007). Es posible, por lo tanto, que las poblaciones de palomas domésticas en Coro no estén consumiendo los hospedadores intermediarios habituales (caracoles).

Se detectaron dos géneros de cestodos, incluyendo Raillietina spp y Hymenolepis spp en frecuencias de 7.8 y $0.2 \%$, respecti- vamente. Las cestodiasis debidas a Raillietina spp son más frecuentes que las ocasionadas por Hymenolepis spp; sin embargo, la ocurrencia de $7.8 \%$ encontrada de Raillietina spp fue más baja que en otros estudios de otros países (Radfar et al., 2011; Bahrami et al., 2012; Diakou et al., 2013).

Mediante la aplicación del Análisis de Correspondencia se observó que la mayoría de los taxones enteroparasitarios identificados en C. livia domestica, especialmente las de mayor ocurrencia, no mostraron una asociación acentuada con los tres sitios en estudio, lo que podría deberse a que esta especie tiene una marcada afinidad por los ambientes. En este mismo sentido, es posible que las poblaciones de palomas de Coro poseen un flujo genético continuo, tal como encontraron Pérez-García et al. (2015) en poblaciones de esta especie en Sincelejo, Colombia, sin poder establecerse una relación entre la variación genética y la distribución espacial.

\section{Conclusiones}

- La ocurrencia de parásitos intestinales en poblaciones ferales de C. livia domestica de la ciudad de Coro, estado Falcón, Venezuela fue de $54.3 \%$.

- Se detectaron hasta tres taxones entero-parasitarios con potencial zoonótico.

\section{Literatura Citada}

1. Abreu-Acuña N, Foronda-Rodríguez P, López M, Valladares B. 2009. Occurrence of Cryptosporidium hominis in pigeons (Columba livia). Acta Parasitol 54: 1-4. doi: 10.2478/ s11686.0008-4

2. Badparva E, Ezatpour B, Azami M, Badparva M. 2015. First report of bird infection by intestinal parasites in Khorramabad, west Iran. J Parasit Dis 39: 720-724. doi: 10.1007/s12639-0140427-5 
3. Badparva E, Ezatpour B, Mahmoudvand $H$, Behzadifar M, Behzadifar M, Kheirandish F. 2017. Prevalence and genotype analysis of Blastocystis hominis in Iran: a systematic review and meta-analysis. Arch Clin Infect Dis 12: e36648. doi: 10.5812/archcid.36648

4. Bahrami AM, Monfared AL, Razmjoo M. 2012. Pathological study of parasitism in racing pigeons: An indication of its effects on community health. Afr J Biotechnol 11: 12364-12370. doi: 10.5897/AJB11.3631

5. Balicka-Ramisz A, Pilarczyk B. 2014. Occurrence of coccidia infection in pigeons in amateur husbandry. Diagnosis and prevention. Ann Parasitol 60: 93-97.

6. Betts EL, Gentekaki E, Thomasz A, Breakell V, Carpenter A, Tsaousis A. 2018. Genetic diversity of Blastocystis in non-primate animals. Parasitology 145 : 122-1234. doi: 10.1017/S0031182017002347

7. Botero D, Restrepo M. 2012. Parasitosis humana. $4^{\mathrm{a}}$ ed. Colombia: Corporación para Investigaciones Biológicas. $733 \mathrm{p}$.

8. Cazorla-Perfetti D, Leal G, Escalona A, Hernández J, Acosta M, Morales $P$. 2014. Aspectos clínicos y epidemiológicos de la infección por coccidios intestinales en Urumaco, estado Falcón, Venezuela. Bol Malar Salud Ambi 54: 159-173.

9. Cazorla-Perfetti D, Lehmann S, Carrero C, Bravo F, Acosta M, Morales P. 2015. Perfiles clínicos y epidemiológicos de la infección por coccidios intestinales en Mirimire, estado Falcón, Venezuela. Saber 27: 37-52.

10. Cazorla D, Morales P. 2013. Prevalencia de parásitos intestinales en gallos de pelea de la ciudad de Coro, estado Falcón, Venezuela. Rev Inv Vet Perú 24: 489-502. doi: 10.15381/rivep.v24i4.2748

11. Cazorla D, Morales P. 2017. Ocurrencia de enteroparásitos en poblaciones de Quiscalus lugubris (Aves: Passeriformes, Icteridae) del semiárido urbano del
Estado Falcón, Venezuela. Rev Inv Vet Perú 28: 178-188. doi: 10.15381/ rivep.v28i1.12941

12. Chacín L, Vielma J. 2018. Ciclosporiasis: distribución, prevalencia y control. Invest Clin 59: 67-93. doi: 10.22209/ ICv.59n1a07

13. Chu DM, Sherchand JB, Cross JH, Orlandi PA. 2004. Detection of Cyclospora cayetanensis in animal fecal isolates from Nepal using an FTA filter-base polymerase chain reaction method. Am J Trop Med Hyg 71: 373379. doi: $10.4269 /$ ajtmh.2004.71.373

14. Copia M, Quiroga D. 2017. Prevalencia de ectoparásitos y endoparásitos en palomas (Columba livia) de plazas y parques en la ciudad de Lambayeque 2016. Tesis de Médico Veterinario. Lambayeque, Perú: Univ. Nacional Pedro Ruiz Gallo. $137 \mathrm{p}$.

15. De La Ossa J, De La Ossa-Lacayo A, Monroy-Pineda M. 2017. Abundance of domestic dove (Columbia livia domestica Gmelin, 1789) in Santiago de Tolú, Sucre, Colombia. Rev MVZ Córdoba 22: 5718-5727. doi: 10.21897/ rmvz.931

16. Diakou A, Ptochos S, Papadopoulos E. 2013. Cestode fauna of feral pigeons in Thessaloniki; Northern Greece. Helminthologia 50: 39-42. doi: 10.2478/ s11687-013-0105-7

17. Ditrich O, Palkovic L, Strba J, Prokopic J, Loudová J, Giboda M. 1991. The first finding of Cryptosporidium baileyi in man. Parasitol Res 77 : 44-47.

18. Díaz-Ungría C.1981. Protozoos de Venezuela. Kasmera 9: 148-215.

19. Faraj A. 2014. Distribution of Cryptosporidium spp infection in wild pigeons in Baghdad city-Iraq. Bas J Vet Res 13: 48-53.

20. Greenacre M. 2008. La práctica del análisis de correspondencias, Manuales Fundación BBVA. [Internet]. Disponible en: http://www.econ.upf.edu/ michael/multivariatestatistics/practica.html 
21. Greige $S$, El Safadi D, Bécu $N$, Gantois N, Pereira B, Chabé M, Benamrouz-Vanneste S, et al. 2018. Prevalence and subtype distribution of Blastocystis sp isolates from poultry in Lebanon and evidence of zoonotic potential. Parasite Vector 11: 389. doi: 10.1186/s13071-018-2975-5

22. Hammer Ø, Harper D, Ryan P. 2001. PAST: Paleontological statistics software package for education and data analysis. Palaeontol Electron 4: 1-9.

23. Hrdlicková J. 2011. Blastocystis u domácího ptactva. Tesis de Maestría. Ceské Budejovice, República Checa: Univ del Sur de Bohemia. $56 \mathrm{p}$.

24. Jasim GA, Marhoon IA. 2015. Prevalence and molecular analysis of Cryptosporidium spp isolated from wild and domestic birds. Acta Parasitologica Globalis 6: 65-70. doi: 10.5829/ idosi.apg.2015.6.2.93253

25. Jayentakumar-Singh L, Mohilal N. 2017. Gastrointestinal parasitic infection in diverse species of domestic birds of Manipur, India. J Parasit Dis 41: 142-146. doi: 10.1007/s12639-016-0767-4

26. Koompapong K, Mori H, Thammasonthijarern N, Prasertbun R, Pintong A, Popruk S, Rojekittikhun W, et al. 2014. Molecular identification of Cryptosporidium spp in seagulls, pigeons, dogs, and cats in Thailand. Parasite 21: 52. doi: 10.1051/parasite/ 2014053

27. Krautwald-Junghanns M, Zebisch R, Schmidt V. 2009. Relevance and treatment of coccidiosis in domestic pigeons (Columba livia forma domestica) with particular emphasis on toltrazuril. J Avian Med Surg 23: 1-5. doi: 10.1647/2007-049R.1

28. Lainson $R$. 2005. The genus Cyclospora (Apicomplexa: Eimeriidae), with a description of Cyclospora schneideri n. sp. in the snake Anilius scytale scytale (Aniliidae) from Amazonian Brazil-a review. Mem I
Oswaldo Cruz 100: 103-110. doi: 10.1590/S0074-02762005000200001

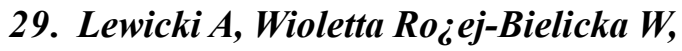
Sa ${ }^{3}$ amatin R. 2016. Blastocystis hominis s. 1. ST6 - parasite of chickens new zoonotic agent in Poland. Ann Parasitol 62: 203.

30. Li J, Lin X, Zhang L, Qi N, Liao S, Lv M, Wu C, Sun M. 2015a. Molecular characterization of Cryptosporidium spp. in domestic pigeons (Columba livia domestica) in Guangdong Province, Southern China. Parasitol Res 114: 22372241. doi: 10.1007/s00436-015-4415-1

31. Li N, Ye J, Arrowood M, Ma J, Wang L, Xu H, Feng Y, Xiao L. 2015 b. Identification and morphologic and molecular characterization of Cyclospora macacae $\mathrm{n}$. $\mathrm{sp}$. from rhesus monkeys in China. Parasitol Res 114: 1811-1816. doi: 10.1007/s00436-0154367-5

32. Marenzoni ML, Morganti G, Moretta I, Crotti S, Agnetti F, Moretti A, Pitzurra L, et al. 2016. Microbiological and parasitological survey of zoonotic agents in apparently healthy feral pigeons. Pol J Vet Sci 19: 309-315. doi: 10.1515/pjvs-2016-0038

33. Morales G Pino de Morales L. 1977. Coprología. En: Manual de diagnóstico helmintológico en rumiantes. Venezuela: Colegio de Médicos Veterinarios del Estado Aragua. p 26-27.

34. Moreno C. 2001. Métodos para medir la biodiversidad. España: M\&T Manuales y Tesis SEA. España: CITED. 84 p.

35. Nagwa EA, El-Akabawy LM, ElMadawy RS, Toulan EI. 2013. Studies on intestinal protozoa of poultry in Gharbia Gobernorate. BENHA Vet Mwed J 25: 78 -83.

36. Oliveira BCM, Ferrari ED, da CruzPanegossi MF, Nakamura AA, Corbucci FS, Nagata WB, Dos Santos BM, et al. 2017. First description of Cryptosporidium parvum in carrier pigeons (Columba livia). Vet Parasitol 243: 148-150. doi: 10.1016/j.vetpar.2017.06 .023 
37. Pazmiño A. 2007. Estudio de la fauna parasitaria intestinal en palomas (Columba livia Gmelin, 1789) de la ciudad de Valdivia. Tesis de Médico Veterinario. Valdivia, Chile: Univ. Austral de Chile. $44 \mathrm{p}$.

38. Pérez-García J, Monsalve-Arcila D, Márquez-Villegas C. 2015. Presencia de parásitos y enterobacterias en palomas ferales (Columba livia) en áreas urbanas en Envigado, Colombia. Rev Fac Nac Salud Pública 33: 370-376. doi: 10.17533/udea.rfnsp.v33n3a06

39. Radfar M, Fathi S, Asl E, Dehaghi M, Seghinsara H. 2011. A survey of parasites of domestic pigeon (Columba livia) in South Khorasan, Iran. Vet Res 4: 18-23. doi: 10.3923/vr.2011.18.23

40. Santín M. 2013. Clinical and subclinical infections with Cryptosporidium in animals. New Zeal Vet J 61: 1-10. doi: 10.1080/00480169.2012.731681

41. Sari B, Karatepe B, Karatepe M, Kara M. 2008. Parasites of domestic (Columba livia domestica) and wild (Columba livia livia) pigeons in Niðde, Turquía. B Vet I Pulawy 52: 551-554.

42. Stensvold C, Clark C. 2016. Current status of Blastocystis: a personal view. Parasitol Int 65: 763-771. doi: 10.1016/ j.parint.2016.05.015
43. Tanveer MK, Kamran A, Abbas M, Umer N, Azhar M, Munir M. 2011. Prevalence and chemo-therapeutical investigations of gastrointestinal nematodes in domestic pigeons in Lahore, Pakistan. Trop Biomed 28: 102-110.

44. Tietz-Marques $S$, De Quadros $R, D a$ Silva C, Baldo M. 2007. Parasites of pigeons (Columba livia) in urban areas of Lages, Southern Brazil. Parasitol Latinoam 62: 183-187. doi: 10.4067/ S0717-77122007000200014

45. Vargas J. 2016. Prevalencia de Cryptosporidium sp en heces de paloma doméstica (Columba livia) de parques y plazas de la ciudad de Huánuco y su efecto en la Salud Pública. Tesis de Maestría. Huánuco, Perú: Univ. de Huánuco. 87 p.

46. Wang R, Qi M, Jingjing Z, Sun D, Ning C, Zhao J, Zhang L, Xiao L. 2011. Prevalence of Cryptosporidium baileyi in ostriches (Struthio camelus) in Zhengzhou, China. Vet Parasitol 175: 151154. doi: 10.1016/j.vetpar.2010.-10.005

47. Wang R, Li J, Chen Y, Zhang L, Xiao L. 2018. Widespread occurrence of Cryptosporidium infections in patients with HIV/AIDS: epidemiology, clinical feature, diagnosis, and therapy. Acta Trop 187: 257-263. doi: 10.1016/j.actatropica.2018.08.018 